\title{
MICRO-/NANO-STRUCTURED ANODES FOR ENHANCED PERFORMANCE OF MICRO-SIZED MICROBIAL FUEL CELLS
}

\author{
A. Fraiwan ${ }^{1}$, S.P. Adusumilli ${ }^{2}$, D. Han ${ }^{3}$, A.J. Steckl ${ }^{3}$, D.F. Call ${ }^{4}$, C.R. Westgate ${ }^{2}$, and S. Choi ${ }^{1 *}$ \\ ${ }^{1}$ Bioelectronics \& Microsystems Laboratory, State University of New York at Binghamton, New York, USA \\ ${ }^{2}$ Center for Autonomous Solar Power, State University of New York at Binghamton, New York, USA \\ ${ }^{3}$ Nanoelectronics Laboratory, University of Cincinnati, Ohio, USA \\ ${ }^{4}$ Environmental Biotechnology Lab, Syracuse University, New York, USA
}

\begin{abstract}
Microbial fuel cells (MFCs) are gaining acceptance as a future alternative green energy technology and energy-efficient wastewater treatment method. Despite their vast potential, however, our ability to harness the potential of MFC technology lags from its low power density limiting its practical applications. Among a number of factors that can affect the MFC's performance, the anode material has the greatest impact on the performance by determining the actual accessible surface area for bacteria to attach and affecting the interfacial electron transfer resistance. In this work, microbial electricity generations on six micro/nanostructured anodes in micro-sized MFCs $(57 \mu \mathrm{L})$ have been investigated by probing the behavior and physiology of microbial biofilm and their interaction with each anode at a new level of detail and efficiency. Six anodes are carbon nanotube (CNT), carbon nanofiber (CNF), gold/PCL microfiber (GPM), gold/PCL nanofiber (GPN), planar gold (PG), and conventional carbon paper (CP).
\end{abstract}

\section{INTRODUCTION}

Microbial fuel cell (MFC) technology is considered to be a promising alternative technology for clean energy production that could help alleviate the global energy crisis and environmental pollution [1]. This has caused an increase in the MFC research focusing on the use of MFCs for renewable energy production. The past few years have witnessed significant developments and performance improvements in MFC technology [2]. These advances are reflected in the increasing number of scientific publications and patents [3, 4]. Despite advances, the promise of the MFC technology has not yet been translated into commercial reality, because existing MFCs demonstrate low power density [5].

Aside from all the other factors affecting the MFC performance such as bacterial inoculums, chemical substrates, and reactor configuration [3], the anode materials play a major role in influencing the power generation by determining: (i) the actual accessible area for bacteria to attach; (ii) the extracellular electron transfer efficiencies; and (iii) chemical species diffusion rates [6-8]. Therefore, many of the studies to date have concentrated on improving anode performance by the search of effective anode material and/or modifications to the anode surface [9-11].

Recently, many unconventional three-dimensional micro/nano-scale and/or micro-fabricated anode materials have been explored to increase surface area, porosity, biocompatibility, conductivity and biofilm formation [7, 9-11]. However, their performances are often more dependent on the specific MFC architecture, electrode spacing, environmental conditions, operating conditions, cathode materials, solution ionic strength and conductivity of the fuel cells. Therefore, power densities produced by a certain anode in one study cannot be directly compared with another anode unless the MFC architecture, bacteria, and chemical solution are the same. In addition, most of these anode studies for improving the MFC anode performances did not provide long-term analyses beyond ten days even though microbial communities and

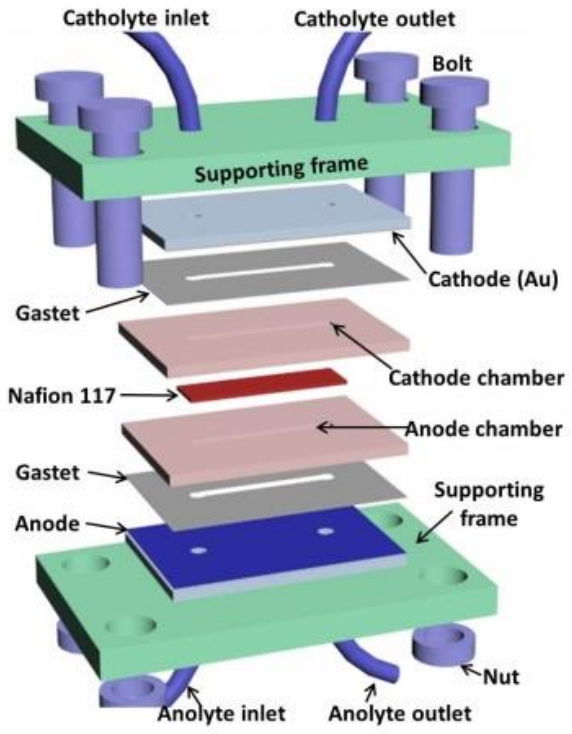

Figure 1: Schematic of the microfabricated MFC. The MFC contains vertically-stacked $57 \mu \mathrm{L}$ anode and cathode chambers separated by a PEM (Nafion 117). Each layer except for the PEM was micro-patterned using laser micromachining.

their electron generating capabilities on anode materials will likely be affected by long-term operation. Therefore, the studies on micro-/nano-structured anode materials reported to date would not provide useful information unless the results are compared in the same condition for a long period of time.

In this paper we compare six popular micro- and nanostructured anode materials in $57 \mu \mathrm{L}$ micro-sized MFCs under the same experimental condition for 1 month. The six anodes are carbon nanotube (CNT), carbon nanofiber (CNF), gold/poly $(\mathcal{E}$ caprolactone) microfiber (GPM), gold/poly(E-caprolactone) nanofiber (GPN), planar gold (PG), and conventional carbon paper (CP). The biofilm profiles, activation loss, internal resistances, and mass transfer losses on each anode material are thoroughly analyzed. The reported results will provide an insightful understanding of the relationship between micro-/nano-structured anodes and active microbial biofilm, which will pave the way for future applications of these novel anode materials in MFC technology.

\section{MATERIALS AND EXPERIMENTAL SET-UP Device Fabrication}

Six micro-sized MFCs were prepared with different anode materials; carbon nanotube (CNT), carbon nanofiber (CNF), gold/poly (E-caprolactone) (PCL) microfiber (GPM), gold/PCL nanofiber (GPN), planar gold (PG), and conventional carbon paper (CP). Each MFC contains vertically-stacked $57 \mu \mathrm{L}$ anode and cathode chambers separated by a PEM (Nafion 117) (Fig. 1). Each 


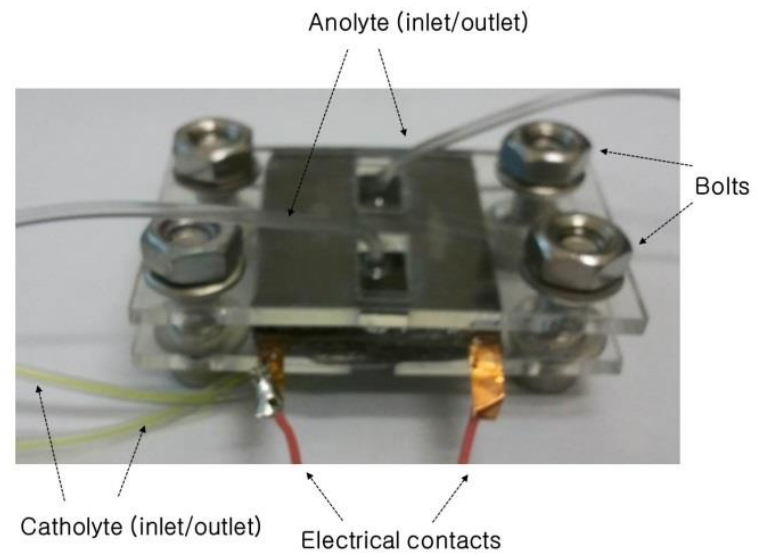

Figure 2: Photography of the fully assembled MFC. All layers were manually stacked in sequence while carefully aligning the tubing holes for the microfluidic channels. 4 tubes were plugged into the holes to form 2 independent routes for anolyte/catholyte access. Copper tape was attached to the contact pads with silver conductive paint.

layer except for the PEM was micro-patterned using laser micromachining (Universal Laser Systems VLS 3.5). Each chamber volume $(4 \mathrm{~cm} \times 3 \mathrm{~cm})$ was defined by $0.158 \mathrm{~cm}$-thick patterned PMMA and $100 \mu \mathrm{m}$-thick thin plastic gasket. The exposed anode/cathode area was $0.28 \mathrm{~cm}^{-2}$. The Nafion 117 was sandwiched by the two PMMA chambers and thermally bonded at $125^{\circ} \mathrm{C}$ for 1 hour. We fabricated $4 \mathrm{~cm} \mathrm{x} 6.2 \mathrm{~cm}$ PMMA supporting frames ( 0.5 inch thick) with the laser and drilled 6 holes for fluidic inlet/outlet and screws. All layers were manually stacked in sequence while carefully aligning the tubing holes for the microfluidic channels. 4 tubes (CODAN, $0.35 \mathrm{~mL}$ volume) were plugged into the holes to form 2 independent routes for anolyte/catholyte access. Copper tape (3MTM copper conductive tape) was attached to the contact pads with silver conductive paint (PELCO® Colloidal Silver) (Fig. 2).

\section{CNT and CNF Fabrication}

Quartz was used as the substrate. A $60 \mathrm{~nm}$ thick aluminum oxide $\left(\mathrm{Al}_{2} \mathrm{O}_{3}\right)$ layer was deposited by atomic layer deposition (ALD) and a 5-15 nm thick Fe layer was deposited by thermal evaporation. The substrate was placed inside the quartz tube in atmospheric pressure chemical vapor deposition (AP-CVD) setup and flushed by argon gas for $30 \mathrm{~min}$. Then the furnace was turned on to the desired temperature $\left(600-800{ }^{\circ} \mathrm{C}\right)$. Hydrogen flow of 10 sccm and argon flow of $200 \mathrm{sccm}$ were passed through the tube. When the temperature reached the set temperature, the flow of ethylene was initiated, and after 2 minutes of ethylene flow, the water molecules were released from the bubbler through argon gas in the range of $150-400 \mathrm{sccm}$. After $60 \mathrm{~min}$ of growth time, the furnace, ethylene flow and water flow were turned off and the furnace was cooled to $20{ }^{\circ} \mathrm{C}$ in $20 \mathrm{~min}$. For carbon nanofibers (CNFs), the synthesis procedure was the same except that the catalyst used is $\mathrm{Ni}$ in the CNF synthesis compared to Fe for CNT synthesis.

\section{GPM and GPN Fabrication}

PCL fibers were prepared by electrospinning, we have used poly $(\mathcal{E}$-caprolactone) (PCL) (Sigma-Aldrich, M.W. $=90 \mathrm{KDa})$ as a fiber material. To fabricate PCL microfiber membranes for the GPM device, the polymer solution dissolving $10 \mathrm{wt} \%$ of PCL in
2,2,2-Trifluoroethanol (TFE) (Acros Organics, 99.8\% purity) solvent was constantly fed at $1.2 \mathrm{~mL} \mathrm{hr}^{-1}$ by a syringe pump. High voltage $\sim 12 \mathrm{kV}$ was applied across a gap of $20 \mathrm{~cm}$ between the needle and the collector. To fabricate PCL nanofiber sample for GPN device without any additives, PCL solution was prepared by dissolving $25 \mathrm{wt} \%$ of PCL into a highly ionic formic acid (Acros Organics, $88 \%$ purity). This solution was fed at $0.1 \mathrm{~mL} \mathrm{hr}^{-1}$ and the high voltage of $24 \mathrm{kV}$ was applied across a gap of $25 \mathrm{~cm}$. Because of very vigorous whipping and stretching actions of the ejected liquid jet, solidified fibers were attached to the collector. After electrospinning, the collected fiber mats were dried in a vacuum oven for $12 \mathrm{hr}$ to remove any residual solvent. For gold coating on PCL electrospun fiber mats, sputtered gold was deposited on fibers for 10 min using Desk II mini-sputter system (Denton).

\section{$\mathrm{CP}$ and PG Fabrication}

Carbon paper (Fuel Cell Store, $0.48 \mathrm{~g} \mathrm{cc}^{-1}, 0.2 \mathrm{~mm}$ ) was cut into $3 \mathrm{~cm} \times 4 \mathrm{~cm}$ pieces and put on the PMMA. The PG was prepared by depositing $100 \mathrm{~nm}$ gold on the PMMA substrate with chrome as the adhesion layer using e-beam evaporation.

\section{Measurement Setup}

We measured the potential between the anode and the cathode by a data acquisition system (NI, USB-6212) and recorded the results every $1 \mathrm{~min}$ via a customized LabVIEW interface. An external resistor was used to close the circuit by connecting the anode and cathode. The current through the load was calculated via Ohm's rule and the output power was calculated via $\mathrm{P}=\mathrm{V} \times \mathrm{I}$. Current and power densities were normalized to the anode area.

\section{Anolyte and Catholyte}

We obtained pre-acclimated anode-respiring bacteria from an acetate-fed MFC initially inoculated with primary clarifier influent and operated for several months. We fed the anode chamber with acetate $\left(1 \mathrm{~g} \mathrm{~L}^{-1}\right.$ in mineral medium) as the sole electron donor. The catholyte was $100 \mathrm{mM}$ ferricyanide in a $100 \mathrm{mM}$ phosphate buffer in which the $\mathrm{pH}$ was adjusted at $7.5 \pm 0.2$ with $0.1 \mathrm{M} \mathrm{NaOH}$.

\section{Bacterial Fixation and SEM Imaging}

The MFCs were disassembled, rinsed, and adherent bacteria on each anode were immediately fixed in $2 \%$ glutaraldehyde solution overnight at $4{ }^{\circ} \mathrm{C}$. Samples were then dehydrated by serial, 5 min transfers through 50,70, 80, 90, 95, and 100\% ethanol. Fixed samples were examined using a FESEM (Supra 55 VP, Zeiss).

\section{RESULTS AND DISCUSSION}

To allow for the accumulation and the acclimation of bacteria on the anode, the anolyte and catholyte were slowly introduced into the anode and cathode chambers. The six MFCs were first operated under open-circuit conditions for 10 days (Fig. 3a). The open-circuit voltage profiles show that there are three different trends corresponding to three stages of biofilm formation: (i) initial bacterial attachment; (ii) biofilm formation through exopolysaccharide substance; (iii) saturation releasing bacteria in the planktonic form and continuing the cycle again. The voltage curves show a significant increase over the first day of operation, followed by a slower increase until the MFCs reached constant voltages at day 7 except for the CNT and CNF anodes whose opencircuit voltages kept increasing even after 10 days of operation which may be attributed to the continuing biofilm formation on the larger surface area. After the initial operation under no load conditions, we measured cell polarization curves and power 

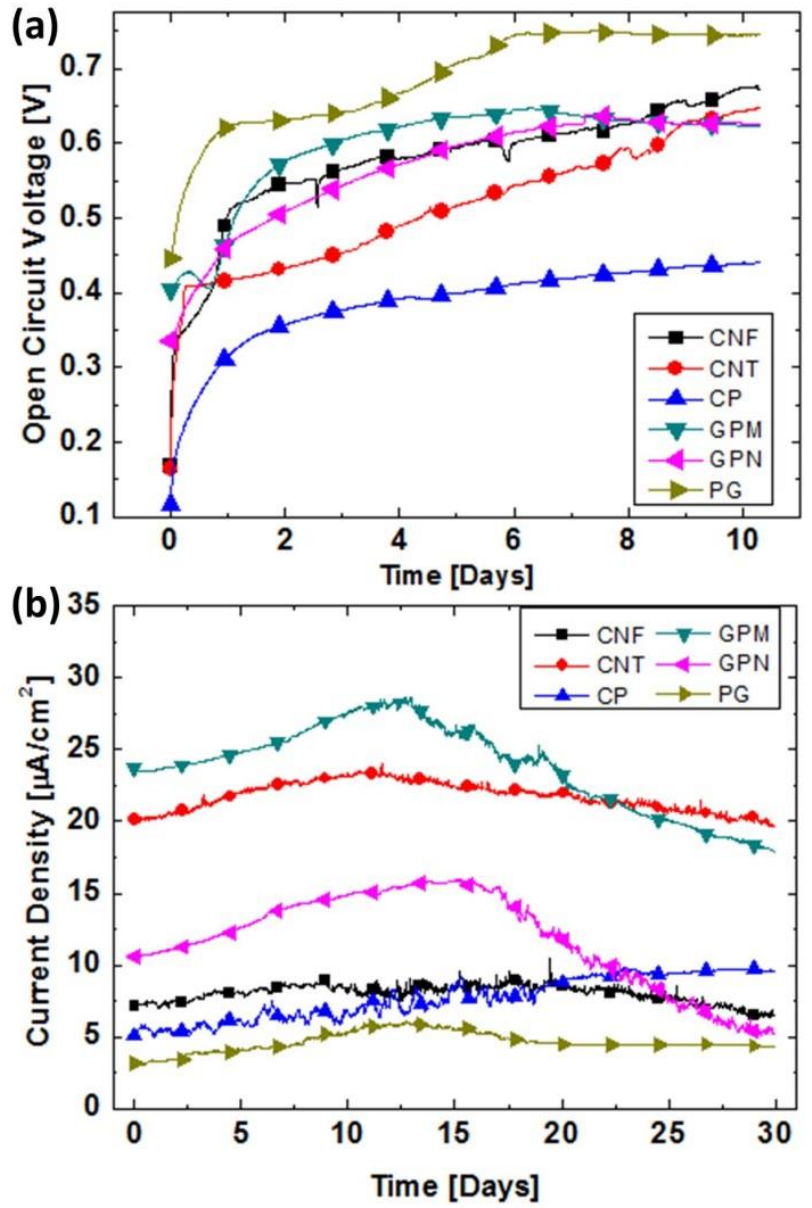

Figure 3: (a) Open circuit voltages and (b) current densities produced form the six MFCs. The maximum open circuit voltages obtained with CNT $(680 \mathrm{mV}), C N F(660 \mathrm{mV}), \mathrm{GPM}$ $(620 \mathrm{mV}), G P N(610 \mathrm{mV})$ and $P G(720 \mathrm{mV})$ were greater than the CP $(400 \mathrm{mV})$. Additional current increase under closed circuit operation indicates the viability recovery of biofilms formed under closed circuit.

outputs for the six cells to obtain the optimal load resistor for maximum power generation for each of the MFCs (Fig. 4). The optimal resistors were used to run the individual MFCs under closed-circuit operation: CNF-22k $\Omega, C N T-10 \mathrm{k} \Omega, \mathrm{CP}-4 \mathrm{k} \Omega$, GPM$10 \mathrm{k} \Omega$, GPN-47k $\Omega$, and PG-100k $\Omega$. The measured current generations under these resistors are shown in Fig. 3b.

The open-circuit voltage curves show that $\mathrm{CP}$ generated the lowest voltage which clearly indicates the substantial energy loss at this anode. Under closed circuit operation, the current density curves showed an additional increase to the values measured in the polarization curves under the same resistor, which is a clear indication of the viability recovery of the biofilms under closedcircuit operation. The biofilm formed in open circuit operation includes an unviable domain in the inner layer of the biofilm because of the inefficient nutrient/oxygen/proton transfer. Switching into closed circuit operation could provide favorable electron acceptor and sufficient energy to support cell growth and metabolisms inside the biofilms, recovering those unviable domains with increases in current. Larger current increases indicate that more unviable regions were recovered. For the longterm operation, $\mathrm{CNT}$ and $\mathrm{CNF}$ anodes reached maximum current densities within approximately 10 days, followed by a slight

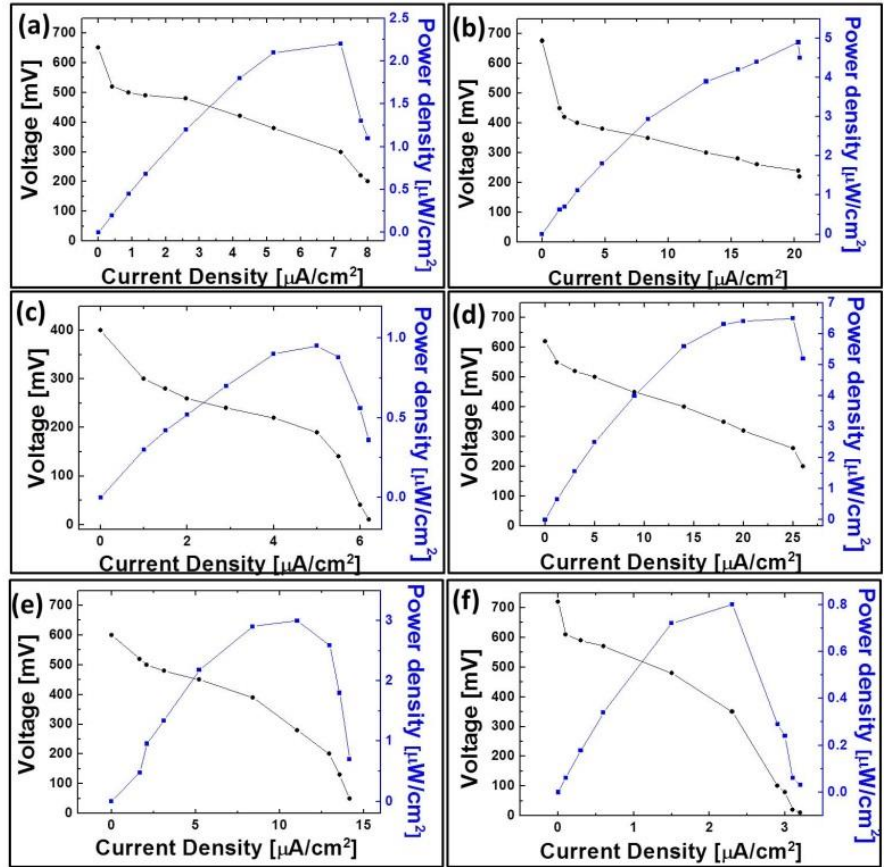

Figure 4: Polarization curve (black circle) and power output (blue square) of the MFCs with six different anode materials, measured as a function of current; (a) CNF, (b) CNT, (c) CP, (d) $G P M$, (e) GPN, and (f) $P G$. The values are derived and calculated based on the maximum current value at a given external resistance (680k, 470k, 330k, 220k, 150k, 100k, 47k, 22k, 10k, and $1 \mathrm{k} \Omega$ ). The individual losses are divided into three zones according to their occurrence at different polarization levels; activation loss, ohmic loss, and mass transfer loss.

Table 1: Overview of the resistivity, OCV, major electrochemical losses, and current densities during closed circuit operations of the MFCs with different anode materials

\begin{tabular}{|c|c|c|c|c|c|}
\hline & $\begin{array}{l}\text { OCV } \\
(\mathrm{mV})\end{array}$ & $\begin{array}{c}\text { Activation } \\
\text { loss } \\
\text { (mV) }\end{array}$ & $\begin{array}{l}\text { Internal } \\
\text { resistance } \\
\quad(\mathbf{k} \Omega)\end{array}$ & $\begin{array}{c}\text { Mass } \\
\text { transfer } \\
\text { loss }(\mathrm{mV})\end{array}$ & $\begin{array}{l}\text { Maximum current } \\
\text { density at optimal } \\
\text { external resistance } \\
\left(\mu \mathrm{A} / \mathrm{cm}^{2}\right)\end{array}$ \\
\hline Carbon fiber (CNF) & 660 & $\sim 130$ & 22 & $\sim 100$ & 8.3 \\
\hline $\begin{array}{l}\text { Carbon nanotube } \\
\text { (CNT) }\end{array}$ & 680 & $\sim 226$ & 12 & $\sim 50$ & 23.4 \\
\hline Carbon paper (CP) & 400 & $\sim 100$ & 30 & $\sim 200$ & 9.6 \\
\hline $\begin{array}{l}\text { Gold/PCL microfiber } \\
\text { (GPM) }\end{array}$ & 620 & $\sim 70$ & 10 & $\sim 60$ & 28.5 \\
\hline $\begin{array}{c}\text { Gold/PCL nanofiber } \\
\text { (GPN) }\end{array}$ & 610 & $\sim 80$ & 23 & $\sim 170$ & 15.8 \\
\hline Planar gold (PG) & 720 & $\sim 90$ & 112 & $\sim 350$ & 5.8 \\
\hline
\end{tabular}

decrease over the next 10 to 15 days. This is likely due to the cellular toxicity of carbon -based nanomaterials that could lead to proliferation inhibition and cell death [12]. GPM and GPN anodes also showed similar current profiles to those of the carbon nanomaterials but with significant current drop from a peak value. The PG anode also experienced current decrease after 12 days.

According to the polarization curves (Fig. 4), three types of electrochemical losses in MFCs could be identified; activation loss, ohmic loss, and mass transfer loss. The values of these losses were calculated for the six MFCs and are given in table 1. Also using 

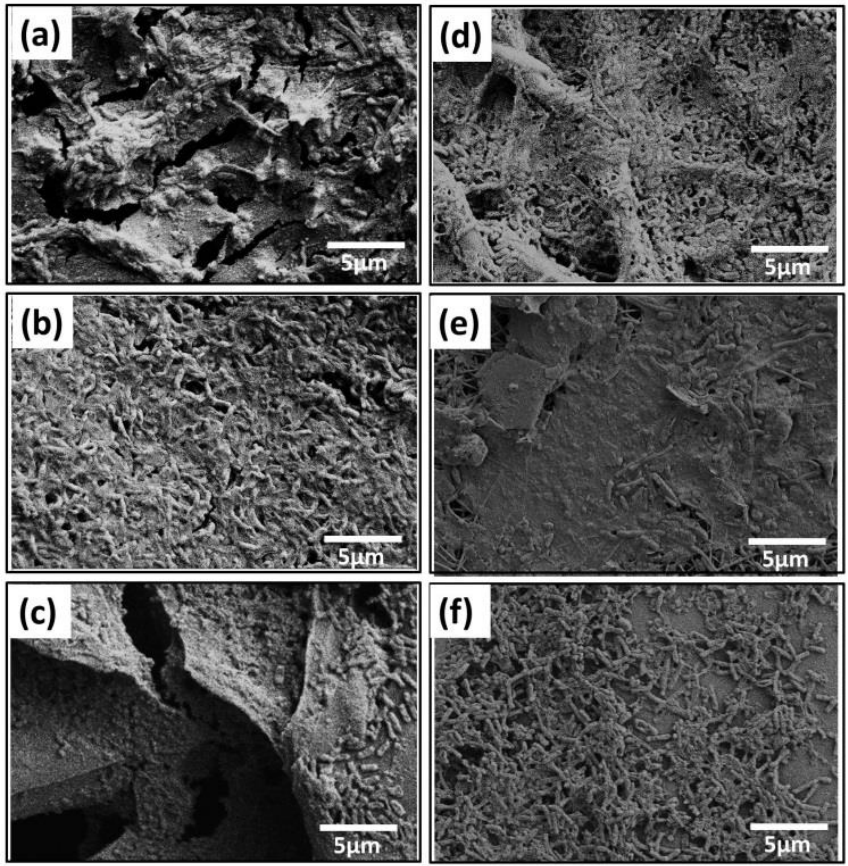

Figure 5: SEM images of all anodes from MFCs after biofilm formation; (a) CNF, (b) CNT, (c) CP, (d) GPM, (e) GPN, and (f) $P G$ (scale bar is shown).

the polarization curve, we estimated the internal resistances from the ohmic loss region by linear fitting of the curve and the resistor values were in good agreement with the external resistor values used for optimal power generation. Carbon-based nanomaterials such as CNT and CNF showed higher activation losses due to the lower conductivity while their mass transfer losses were relatively less than others. The PG-based MFC showed the highest internal resistance $(112 \mathrm{k} \Omega)$, while other three-dimensional micro-/nanoanodes had lower resistances of $10 \sim 30 \mathrm{k} \Omega$, which demonstrates the ability of three dimensional anodes in decreasing the MFC internal resistance. Micro-sized MFCs generally have high internal resistances due to inefficient proton transfer rate [13]. Therefore, three dimensional anodes can allow a more efficient proton flux through the biofilm and a subsequent decrease in the internal resistance.

Finally, SEM images of all anodes from the six MFCs were taken after biofilm formation (Fig. 5) in order to observe the presence of attached biofilms on the different anode materials. In general, the images indicate that biofilms are fully formed on each anode surface, displaying rough uneven topography. On the other hand, the image of the planar gold (PG) surface showed that there is not complete coverage with bacterial cells, which suggests that the biofilm formation on the PG surface was slower than the other materials or that gold is not an optimal surface for bacterial cell attachment. The images also provide good comparative information demonstrating clear differences in the architectures of the biofilm established on the anode surface and the MFC performances.

\section{CONCLCUSION}

This work provides a direct comparison of the performance of six high-surface area anode materials under identical experimental conditions. The MFCs using three dimensional anode structures (CNT, CNF, GPM, and GPN) exhibited lower internal resistances than the macroscopic CP and two-dimensional PG anodes. However, those novel anode materials suffered from major issues such as high activation loss and instability for long-term operation, causing an enduring problem in creating widespread commercial MFC applications. The reported work provides an in-depth understanding of the interplay between micro-/nano-structured anodes and active microbial biofilm, suggesting future directions of those novel anode materials for MFC technologies.

\section{ACKNOWLEDGMENT}

We thank the Nanofabrication Lab at SUNY-Binghamton for providing the fabrication facilities, and the Binghamton University Research Foundation for funding this work. This work was also supported in part by $\mathrm{S}^{3}$ IP Small Grant Award.

\section{REFERENCES}

[1] B. E. Rittmann, "Opportunities for Renewable bioenergy using microrganisms", Biotechnol. Bioeng., 100, 203 (2008).

[2] D. R. Lovley, "Electromicrobiology", Annu. Rev. Microbiol., 66, 391 (2012).

[3] B. E. Logan, and J. M. Regan, "Microbial Fuel CellsChallenges and Applications", Environ. Sci. Technol., 40, 5172 (2006).

[4] J. C. Biffinger, and B. R. Ringeisen, "Engineering Microbial Fuel Cells: Recent Patents and New Directions", Recent Pat. Biotechnol., 2, 150 (2008).

[5] B. E. Logan, "Scaling Up Microbial Fuel Cells and Other Bioelectrochemical Systems", Appl. Microbiol. Biotechnol., 85, 1665 (2010).

[6] V. Flexer, J. Chen, B. C. Donose, P. Sherrell, G. G. Wallace, and J. Keller, "The Nanostructure of Three-Dimensional Scaffolds Enhances the Current Density of Microbial Bioelectrochemical Systems", Energy Environ., Sci., 6, 1291 (2013).

[7] X. Xie, G. Yu, N. Liu, Z. Bao, C. S. Criddle, and Y. Cui, "Graphene-Sponges as High-Performance Low-Cost Anodes for Microbial Fuel Cells", Energy Environ. Sci., 5, 6862 (2012).

[8] J. E. Mink, and M. M. Hussain, "Sustainable Design of High Performance Micro-sized Microbial Fuel Cell with Carbon Nanotube Anode and Air Cathode", ACS Nano, 7, 6921 (2013).

[9] H. Ren, and J. Chae, "Scaling Effect on MEMS-Based Microbial Fuel Cells: Toward a Carbon-Neutral Miniaturized Power Source", Technical Digest of the 2012 Solid-state Sensors Actuators and Microsystems Workshop, Hilton Head Island, SC, 6/3-7/2012, pp. 501-504.

[10] V. Jayaprakash, R.D. Sochol, R.Warren, A. Kozinda, K. Iwai, and L. Lin, "Stackable Cow Dung Based Microfabricated Microbial Fuel Cells," Proceedings of 26th IEEE Micro Electro Mechanical Systems Conference, Taipei, Taiwan, 1/2024/2013, pp. 881-884.

[11] A. Fraiwan, S. Sundermier, D. Han, A. Steckl, D.J. Hassett, and S. Choi, "Enhanced Performance of MEMS Microbial Fuel Cells Using Electrospun microfibrous Anode and Optimizing Operation," Fuel Cells, 13, 336 (2013).

[12] M. Ghasemi, W. R. W. Daud, S. H. A. Hassan, S. Oh, M. Ismail, M. Rahimnejad, and J. M. Jahim, J. Alloys Compd., "Nano-Structured Carbon as Electrode Material in Microbial Fuel Cells: A Comprehensive Review”, 580, 245 (2013).

[13] R .S. Renslow, J. T. Babauta, P. D. Majors, and H. Beyenal, "Diffusion in Biofilms Respiring on Electrodes", Energy Environ. Sci., 6, 595 (2013).

\section{CONTACT}

*S. Choi, tel: +1-607-777-5913; sechoi@ binghamton.edu 